\title{
The expression of DNA methyltransferases3A is specifically downregulated in chorionic villi of early embryo growth arrest cases
}

\author{
HUATING GU ${ }^{1}$, JING GAO ${ }^{2}$, WEIWEI GUO ${ }^{3}$, YU ZHOU $^{1}$ and QINGNUAN KONG $^{4}$ \\ ${ }^{1}$ Department of Physiology and Pathophysiology, Medical College of Qingdao University, Qingdao, \\ Shandong 266071; ${ }^{2}$ Department of Reproductive Medicine Center, Qingdao Municipal Hospital, \\ Affiliated to Medical College of Qingdao University, Qingdao, Shandong 266011; ${ }^{3}$ Department of Pathology, \\ Yantaishan Hospital, Affiliated to Medical College of Qingdao University, Yantai, Shandong 264001; \\ ${ }^{4}$ Department of Pathology, Qingdao Municipal Hospital, Affiliated to Medical College of Qingdao University, \\ Qingdao, Shandong 266011, P.R. China
}

Received April 9, 2016; Accepted March 14, 2017

DOI: $10.3892 / \mathrm{mmr} .2017 .6650$

\begin{abstract}
The aim of the present study was to investigate the expression pattern of four DNA methyltransferases (DNMT1, DNMT3A, DNMT3B and DNMT3L) in placenta chorionic villi of early embryo growth arrest patients. Chorionic villous specimens were obtained from 40 pregnant patients diagnosed with early embryo growth arrest and 40 healthy women who underwent selective pregnancy termination. Reverse transcription-quantitative polymerase chain reaction, immunohistochemistry and western blot analysis were performed to characterize the mRNA and protein expression of DNMTs in chorionic villous cells. It was identified, among the four DNMTs, DNMT3B presented the highest level of protein expression in both patient groups. Although the mRNA expressions of the four DNMTs were comparable, the DNMT3A protein was specifically downregulated in patients with early embryo growth arrest. Therefore, the current study suggests that an abnormal decrease in DNMT3A protein levels may be involved in the pathogenesis of early embryo growth arrest.
\end{abstract}

Correspondence to: Dr Qingnuan Kong, Department of Pathology, Qingdao Municipal Hospital, Affiliated to Medical College of Qingdao University, 1 Jiaozhou Road, Qingdao, Shandong 266011, P.R. China

E-mail: nuanyun4621@126.com

Abbreviations: DNMT, DNA methyltransferase; IUGR, intrauterine growth restriction; ICF syndrome, immunodeficiency-centromeric instability-facial anomalies syndrome

Key words: DNA methyltransferases, early embryo growth arrest, placenta, chorionic villi, epigenetic modulation

\section{Introduction}

Early embryo growth arrest is a unique form of spontaneous abortion, which is characterized by absent fetal development or fetal death in the first-trimester of pregnancy $(1,2)$. With the environmental pollution and increasing life stress in populations, a previous epidemiological study demonstrated that early embryo growth arrest occurs in $>4 \%$ of pregnant women in China and the incidence is increasing rapidly (3). Although a number of factors, such as genetic abnormality, immune system deficits and viral infection have been indicated in the pathogenesis of early growth arrest, the underlying cellular and molecular mechanisms remain poorly understood. Furthermore, although $~ 50 \%$ of incidence of early embryo growth arrest can be ascribed to chromosome abnormality, the etiology of the other clinical cases remains ambiguous $(3,4)$.

The placenta functions as the exchange organ between the fetal and maternal bodies, and it serves a major role in providing nutrients and carrying over metabolic substrates via trans-placental exchange. Fetal development and pregnancy maintenance are dependent on normal placental growth (5). In mammals, epigenetic modification is commonly overlaid during embryogenesis and early development of the fetus. DNA methylation is the most important epigenetic regulation process in genomic imprinting, transposon silencing, $\mathrm{X}$-inactivation and gene repression (6-8). DNA methylation is regulated by DNA methyltransferases (DNMTs), including DNMT1, DNMT3A, DNMT3B and DNMT3L. The dynamics of DNA methylation is vital for embryonic and placental development in physiological and pathological conditions (9-12).

The threemainDNMTs(DNMT1,DNMT3AandDNMT3B) are responsible for two special methylation processes that are required for tissue-specific methylation patterns. DNMT1 mediates maintenance DNA methylation, while DNMT3A and DNMT3B are responsible for de novo methylation $(7,13)$. $D N M T 3 L$ is highly homologous to DNMT3A and DNMT3B in sequence, but has no catalytic activity in vitro. Previous studies have revealed that mutation or deletion of DNMT1, DNMT3A 
and DNMT3B triggered cell death in human embryonic stem cells $(12,14)$. At present however, little is known about the patterns of DNA methylation or DNMTs expression in human placenta during early pregnancy. Some previous studies have reported low expression of 5-methyl-cytosine and relative hypo-methylation in the developmental placenta of different pregnancy stages (15). Previous results based on gene knockout experiments indicated that all the four DNMTs are involved in human trans-acting imprinting defects. For instance, DNMT3B gene mutations of specific residues in the $\mathrm{C}$-terminal catalytic domain are known to cause immunodeficiency-centromeric instability-facial anomalies syndrome $(16,17)$. Aberrant expression of DNMTs may be responsible for the high rate of abortion and abnormal embryo growth. In addition, DNMT3a or DNMT3b knockout mice exhibited embryonic development arrest because of the failure to initiate de novo methylation following implantation $(18,19)$. In addition, wide demethylation and developmental arrest was reported in DNMT1 knockout mice embryos at the early stage of gestation (20).

To test the involvement of abnormal DNA methyltransferases in early embryo growth arrest, the authors measured the level of DNMTs expression in chorionic villi obtained from pregnant women diagnosed with early embryo growth arrest and control patients. A significant downregulation of the DNMT3A protein was observed in these embryo growth arrest cases; however, their DNMT3a mRNA expression levels were comparable to the controls. The discordance of the mRNA and protein expression of DNMT3A indicated that the translation process of DNMT3A was specifically interfered during early embryo growth arrest.

\section{Materials and methods}

Clinical data and tissue samples. Chorionic villous samples were obtained from 80 pregnant women (gestational age ranging from 6 to 9 weeks) who underwent termination of pregnancy at the Department of Obstetrics in the Qingdao Municipal Hospital between January 2013 and June 2014. A total of 40 of the 80 cases were diagnosed with embryo growth arrest by B-mode ultrasound; the other 40 cases were having a normal pregnancy, but patients had voluntarily requested for the termination of pregnancy. Patient age was between 22 and 34 years old, and other serious medical conditions were ruled out, such as chromosome abnormities, endocrine diseases, infection, immunological diseases and other serious maternal complications. Each sample ( $20 \mathrm{~g})$ was immediately frozen in liquid nitrogen for $30 \mathrm{~min}$, and subsequently stored at $-80^{\circ} \mathrm{C}$ for reverse transcription-quantitative polymerase chain reaction (RT-qPCR) and western blot analysis. The remainder of each sample was fixed with $10 \%$ buffered formalin and was paraffin-embedded for immunohistochemistry. Written informed consent for the publication of any associated data and accompanying images was obtained from all patients involved. The current study was approved by the Ethical Review Committee of Qingdao University (Qingdao, Shandong, China).

RNA extraction, cDNA synthesis and RT-qPCR. Total RNA was extracted from the chorionic villi with a PureLink ${ }^{\mathrm{TM}}$ RNA Mini kit (Thermo Fisher Scientific, Inc., Waltham, MA, USA), according to the manufacturer's instructions. RNA quantity and quality was determined by a NanoDrop 2000 spectrophotometer (Thermo Fisher Scientific, Inc., Wilmington, DE, USA). cDNA was then synthesized from $1 \mu \mathrm{g}$ total RNA with SuperScript $^{\mathrm{TM}}$ III Reverse transcriptase (Thermo Fisher Scientific, Inc.) according to the manufacturer's instructions. The mRNA expressions of DNMTs and $\beta$-actin were measured by the Master Cycler ep realplex PCR system (Eppendorf, Hamburg, Germany) using a QuantiFast SYBR-Green PCR kit (Qiagen GmbH, Hilden, Germany) according to the manufacturer's protocols. The PCR cycling parameters were set as follows: $95^{\circ} \mathrm{C}$ for $5 \mathrm{~min}$, followed by 40 cycles of PCR reaction at $95^{\circ} \mathrm{C}$ for $5 \mathrm{sec}$, and finally $60^{\circ} \mathrm{C}$ for $30 \mathrm{sec} . \beta$-actin was used as an internal control. All reactions were run in triplicate. The threshold cycle is defined as the fractional cycle number at which the fluorescence passes the fixed threshold. The data were obtained by normalizing DNMT1, DNMT3a, DNMT3b and DNMT3L genes threshold values with corresponding $\beta$-actin threshold value, and then analyzed with $2^{-\Delta \Delta C q}$ method (21). The primer sequences are presented in Table I.

Immunohistochemical staining and analysis. The biopsied tissues were initially fixed in $10 \%$ buffered formalin, embedded in paraffin and cut into $4 \mu \mathrm{m}$ sections. Tissue sections were then de-paraffinized and dehydrated in graded ethanol and dimethylbenzene, respectively. Antigen retrieval was performed by boiling sections in EDTA buffer ( $\mathrm{pH} 8.0$ ) for $2.5 \mathrm{~min}$ in a pressure cooker. The endogenous peroxidase activity was blocked using $0.3 \%$ hydrogen peroxide. After rinsing in phosphate-buffered saline (PBS), the sections were incubated for $1 \mathrm{~h}$ with polyclonal rabbit anti-DNMT1 antibody (1:200, NB100-264, Novus Biologicals, LLC, Littleton, CO, USA), polyclonal rabbit anti-DNMT3A antibody (1:400, NB100-265, Novus Biologicals, LLC), polyclonal rabbit anti-DNMT3B antibody (1:800, NB100-266, Novus Biologicals, LLC) or polyclonal rabbit anti-DNMT3L antibody (1:100, ab115522, Abcam, Cambridge, UK). After rinse in PBS for several times, the sections were incubated with a biotinylated goat anti-rabbit secondary antibody (UltraSensitive ${ }^{\mathrm{TM}}$ SP kit, Fuzhou Maixin Biotech Co., Ltd., Fuzhou, China) at $37^{\circ} \mathrm{C}$ for $30 \mathrm{~min}$. Sections were then stained using 3,3-diaminobenzidine chromogen solution (Zhongshan Golden Bridge Biotechnology Co., Ltd., Beijing, China) and counterstained with hematoxylin (Fuzhou Maixin Biotech Co., Ltd). All slides were observed separately by two pathologists.

Western blotting detection of DNMTs. The frozen tissue was re-suspended in passive lysis buffer (Promega Corporation, Madison, WI, USA) including freshly added protease inhibitors and phosphatase inhibitors cocktails (Sigma-Aldrich, Darmstadt, Germany). Protein concentrations in the supernatant fractions were determined using a bicinchoninic acid assay kit (Thermo Fisher Scientific, Inc.). Protein lysates were separated with 8 or $12 \%$ SDS-acrylamide gels, and transferred onto nitrocellulose membranes. After blocking for $60 \mathrm{~min}$ in a $5 \%$ skim milk solution, membranes were incubated overnight at $4^{\circ} \mathrm{C}$ with monoclonal rabbit anti-DNMT1 antibody at 1:1,000 (D59A4, Cell Signaling Technology, Inc., Danvers, MA, USA), monoclonal rabbit anti-DNMT3A at 1:1,000 (D23G1, Cell Signaling Technology, Inc.) and polyclonal rabbit 
Table I. Sequences of specific gene primers used for reverse transcription-quantitative polymerase chain reaction.

\begin{tabular}{llc} 
Gene & Forward sequence (5'-3') & Reverse sequence (5'-3') \\
\hline$\beta$-actin & CACTCTTCCAGCCTTCCTTC & GTACAGGTCTTTGCGGATGT \\
DNMT1 & GACCCGTCTCTTGAAGGTGG & CTTCTCCTGCATCAGCCCAA \\
DNMT3A & GGTCACGCAAAACAGAACCC & CCTTGGTGAAACCCTTTGCG \\
DNMT3B & CTCTTCCTCAGCTGTGTGGG & CTGTCGGCACTGTGGTTTG \\
DNMT3L & GATTCTTTGCCCCCATAGCCT & TTCAAGGTTCCAGTGGTCCG
\end{tabular}

anti-DNMT3B at 1:1,000 (NB100-266, Novus Biologicals, LLC), polyclonal rabbit anti-DNMT3L at 1:1,000 (ab115522, Abcam) or monoclonal mouse anti- $\beta$-actin at 1:5,000 (A5441, Sigma-Aldrich). Signals from horseradish-peroxidase-conjugated secondary antibodies were visualized by enhanced chemilluminescence solution (GE Healthcare Life Sciences, Chalfont, UK) and processed with an UVP visualizer (UVP, Inc., Upland, CA, USA). Quantification was performed using VisionWorks LS version 7.0 from the UVP visualizer itself. Experiments were repeated in triplicate.

Statistical analysis. The statistical analysis was performed using SPSS version 17.0 software (SPSS Inc., Chicago, IL, USA). Student's t test was used to compare the quantitative data between the two groups and a one-way analysis of variance (ANOVA) followed by Bonferroni post hoc test was used for multiple comparisons. The results are presented as the mean \pm standard error of the mean and a two-sided $\mathrm{P}<0.05$ was considered to indicate a statistically significant difference.

\section{Results}

DNMT mRNA expression in chorionic villi of early embryo growth arrest patients. First, the authors measured the level of mRNA expression of DNMT1, DNMT3A, DNMT3B and DNMT3L in chorionic villi obtained from the placental tissue of both groups. DNMT3B presented the highest level of expression compared to other three DNMTs in both groups (One-way ANOVA, $n=40$ for each group, $\mathrm{P}<0.0001$, Fig. 1), indicating that DNMT3B is the major type of DNA methyltransferase expressed in chorionic villi. In addition, it was identified that the mRNA expression of four DNMTs (DNMT1, DNMT3A, DNMT3B and DNMT3L) in chorionic villi of early embryo growth arrest patients were comparable to that in the controls, respectively (unpaired Student's t-test, $n=40$ for each group, $\mathrm{P}>0.05$, Fig. 1).

DNMT protein expression in chorionic villi of early embryo growth arrest patients by IHC. Next, IHC studies were conducted to determine whether the protein expression of DNMTs was altered in patients with early embryo growth arrest. IHC staining demonstrated that all four types of DNMTs were expressed in trophoblast cells and chorionic villi. DNMT3A and DNMT3B positive staining was localized in the cell nucleus (Fig. 2A) while DNMT1and DNMT3L were in the cytoplasm (data not shown). Immunostaining intensity was calculated by Image Pro plus 6.0 analysis software (Media

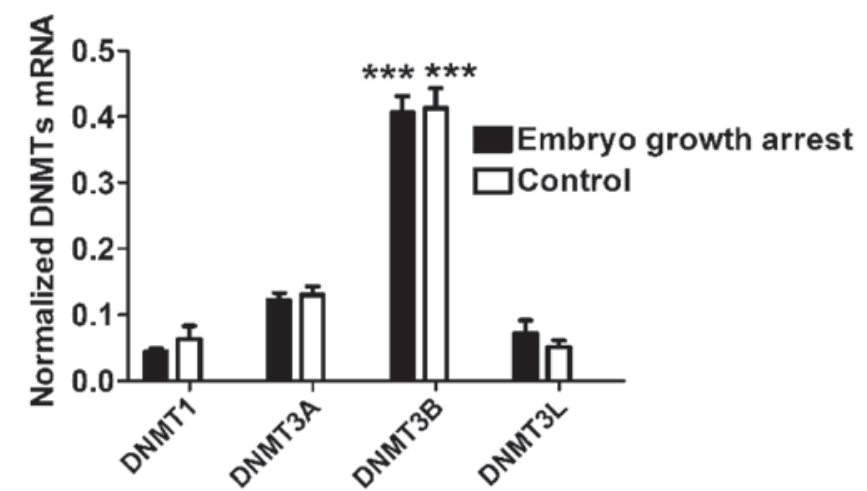

Figure 1. Relative mRNA expression of DNMTs in chorionic villi of early embryo growth arrest patients and normal controls. The mRNA expression was detected using reverse trnascription-quantitative polymerase chain reaction. Data were normalized by $\beta$-actin internal control. The levels of DNMT1, DNMT3A, DNMT3B and DNMT3L mRNA from early embryo growth arrest patients have no significant changes compared to those of normal controls. The data are presented as means \pm standard error of the mean, $\mathrm{n}=40$ for each group, ${ }^{* * *} \mathrm{P}<0.001$ vs. all other DNMT groups. DNMT, DNA methyltransferase.

Cybernetics Inc., Rockville, MD, USA) and represented with semi-quantitative integrated optical density (IOD). The total IOD value of each sample was calculated by summing up the staining intensity from six randomly selected visions per section and across six representative sections. The current results revealed that the expression of DNMT3A protein in chorionic villi of early embryo growth arrest patients was significantly lower than that in chorionic villi of the controls (unpaired Student's t-test, $\mathrm{n}=40$ for each group, $\mathrm{P}<0.01$, Fig. $2 \mathrm{~B}$ ). The protein levels of DNMT1, DNMT3B and DNMT3L were similar between two groups (unpaired Student's t test, $n=40$ per group, $\mathrm{P}>0.05$, Fig. 2B).

DNMTs protein expression in chorionic villi of early embryo growth arrest patients by western blotting. To confirm the above results, western blot analysis was conducted to compare the DNMT expression in chorionic villi from patients with early embryo growth arrest with healthy controls. Data were analyzed with the UVP gel imaging system. Consistently, it was identified that, in comparison to the controls, DNMT3A protein expression was specifically decreased in chorionic villi obtained from embryo growth arrest patients (unpaired Student's t-test, $\mathrm{n}=40$ per group, $\mathrm{P}<0.05$, Fig. $3 \mathrm{~B}$ ). No significant difference was presented between groups as for DNMT1, DNMT3B and DNMT3L expression (unpaired Student's t-test, $\mathrm{n}=40$ per group, $\mathrm{P}>0.05$, Fig. 3 ). 

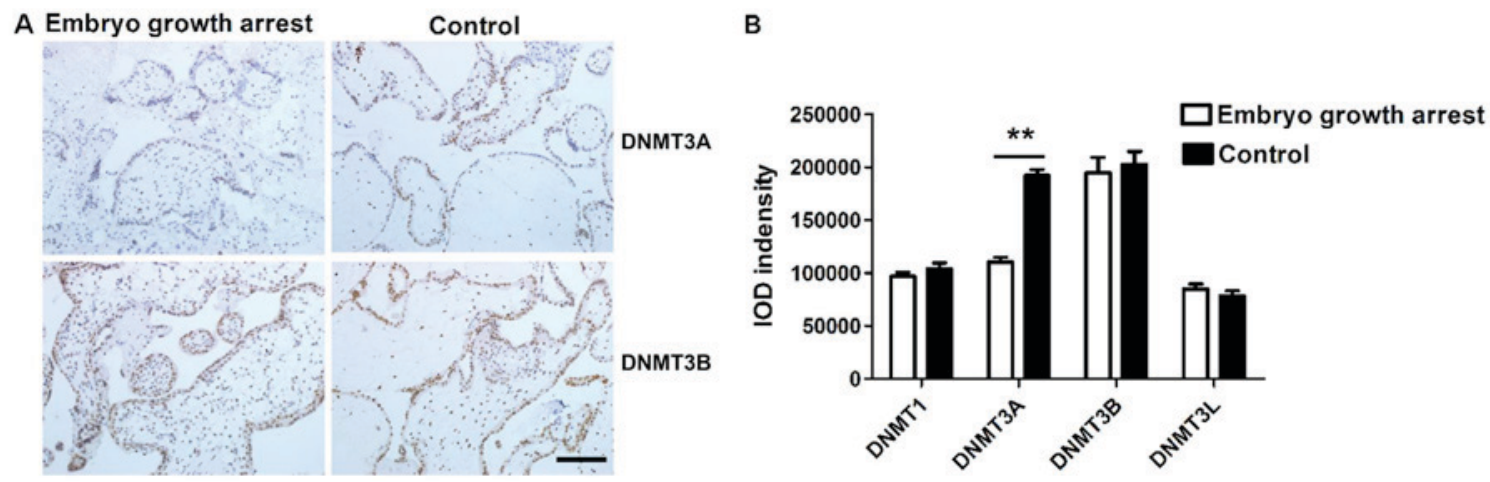

Figure 2. Semi-quantitative analysis of DNMT protein expression in chorionic villous cells by immunohistochemistry. (A) Representative results of immunohistochemistry (magnification, x200). The paraffin-embedded placental tissues were sliced into $4 \mu \mathrm{m}$ sections. The nuclei were stained blue with haematoxylin. DNMT3A and DNMT3B proteins were stained brown and were localized mostly in the nucleus of trophoblast cells. Scale bar, $50 \mu \mathrm{m}$. (B) Histogram indicated a lower IOD (integrated optical density) value of DNMT3A in chorionic villous cells from embryo growth arrest patients, when compared to that from controls. The data are presented as means \pm standard error of the mean, $\mathrm{n}=40$ per group, ${ }^{* * *} \mathrm{P}<0.01$ vs. control. DNMT, DNA methyltransferase.
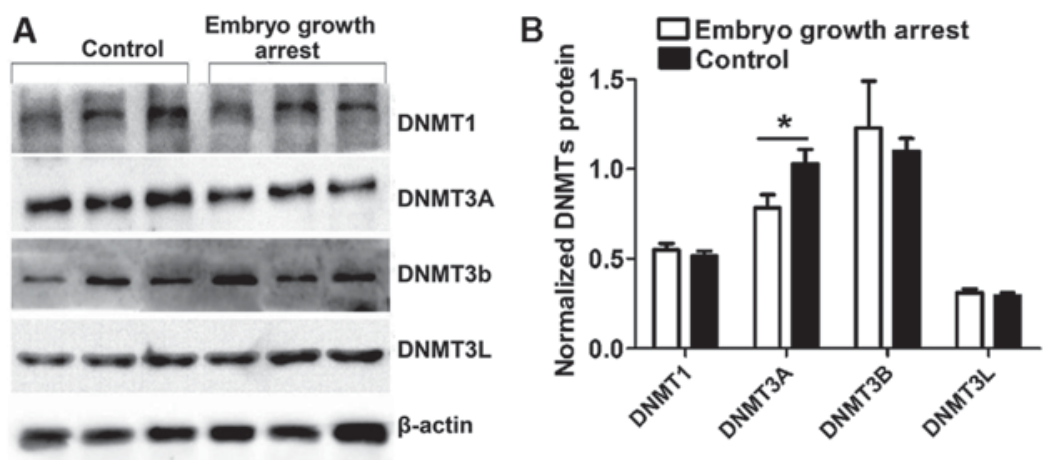

Figure 3. Western blot analysis presented DNMT expression in the chorionic villi of embryo growth arrest patients and controls. (A) Representative blots presenting DNMT expression in chorionic villi. The expression of $\beta$-actin served as an internal control. (B) Histogram reporting reduced DNMT3A protein expression in the embryo growth arrest group. The amount of DNMTs proteins were normalized by $\beta$-actin. The data are presented as means \pm standard error of the mean, $\mathrm{n}=40$ per group, ${ }^{\mathrm{P}} \mathrm{P}<0.01$ vs. control. DNMT, DNA methyltransferase.

\section{Discussion}

In recent years, serious pregnancy complications, such as early embryo growth arrest, recurrent spontaneous miscarriage, intrauterine growth restriction and preeclampsia, have globally been on the increase despite improved medical environment $(1,22)$. These complications are the leading causes of mortality and they put huge emotional and financial burdens on the caregivers and patients themselves. Early embryo growth arrest is a stop in intrauterine embryonic development in the early period of pregnancy (1). Distinct from spontaneous abortion, the majority of the pregnant women with early embryo growth arrest present no obvious symptoms, such as bleeding or abdominal pain $(1,2)$. Early diagnosis of embryo growth arrest primarily depends on B-mode and Doppler ultrasound examination (23). Although previous etiology studies have focused on chromosome abnormality $(1,24-26)$, sperm deformity, immune factors, environment factors and hormonal regulation, the molecular mechanisms of embryo growth arrest remain uncertain.

DNA methylation is crucial during the entire embryonic development. Specifically, it is reported to be important in various cellular processes such as cell differentiation, gene transcription and genomic imprinting $(10,11)$. DNA methylation is established in epiblast cells within embryonic days 4.5 and 6.5 (12). Previous studies indicated that genomic imprinting, regulated by DNA methylation, may serve a critical role in placenta growth $(27,28)$. Methylation levels of human placenta increase in a gestational stage-dependent manner (26). In addition, recent studies demonstrated the association between changes of placental DNA methylation patterns and pregnancy disorders (29). Furthermore, dynamic regulation of DNA methylation by DNMTs exists in placenta $(11,12)$. Therefore, understanding the role and underlying mechanisms of DNA methylation in placental development may offer a new strategy to prevent serious pregnancy complications.

In the present study, the authors investigated the expression patterns of four DNMTs (DNMT1, DNMT3A, DNMT3B and DNMT3L) in chorionic villi of pregnant women and demonstrated that all four DNMTs were expressed in placental villi and trophoblast cells. The expression levels of DNMT3B mRNA and protein were the highest both in patients with early embryo growth arrest and in individuals with normal pregnancy, suggesting that DNMT3B may be one of the prominent DNA methyltransferases involved in placenta development during early pregnancy. More importantly, 
specific downregulation of the DNMT3A protein was observed with both immunostaining and western blot analysis in the chorionic villi of pregnant women with embryo growth arrest, while none of other three DNMTs (DNMT1, DNMT3B or DNMT3L) was changed at both mRNA and protein levels. To the best of the authors' knowledge, the maintenance DNA methylation is a crucial and indispensable element in embryo implantation and development process $(10,11)$. Previous studies suggested that both DNMT3A and DNMT3B are essential for de novo methylation in the embryonic stem cells and early embryos (30). In a mouse model, the DNMT3B protein is more enriched in the embryonic stem cells than DNMT3A $(9,19)$. However, the DNMT3A protein begins to be expressed again in the middle embryonic development (E10.5-E14.5), while DNMT3B is not (9). These studies support the theory that epigenetic alterations in early embryos may be carried forward to subsequent developmental stages (31). Moreover, DNMT3A transcription is presumably the vital point for embryonic development process. Therefore, the finding that an abnormal decrease of chorionic villous DNMT3A protein in the placenta of early embryo growth arrest patients may be important for the pathogenesis of early embryo growth arrest. Decreased protein expression accompanied by normal mRNA expression of DNMT3A also indicated that DNMT3A protein translation or post-translation processing was specifically interfered in early embryo growth arrest.

The placenta serves an important role in managing intrauterine embryonic growth and development through nutrients and waste transfer (32). Previous studies have indicated that placental epigenetic profiles may affect fetal growth through the interaction between intra-uterine and extra-uterine environments (33-35). Administration of DNA methyltransferase inhibitors to pregnant rats resulted in smaller placentas with severe histological damage at different gestational ages (36). Another previous study reported that DNMT3B mRNA expression decreases following the increase in DNMT3A and DNMT1 expression in early pregnancy placenta (37). Other studies indicated that the DNMT1 protein level was significantly decreased and the global DNA methylation level was significantly downregulated in chorionic villi of women with early pregnancy loss $(27,29)$. Although different subtypes of DNMTs were reported to be downregulated in the present study compared to the previous reports, the downregulation trends of DNMTs expression was the same. Our findings indicated that the downregulation of DNMT3A expression in chorionic villi is specific in early embryo growth arrest. Insufficient maintenance methylation due to the lower level of DNMT3A protein expression may be associated with abnormal embryonic development in human early pregnancy loss.

In summary, DNA methylation has a critical role in placenta development, and changes in methylation pattern can lead to adverse birth outcome. Placental epigenome may serve as a modulator of disease pathogenesis. The current findings confirmed that downregulation of DNMT3A protein expression and the ensuing disturbance of the maintenance DNA methylation may serve an important role in the pathogenesis of early embryo growth arrest. Further studies are required to disclose the specific target genes regulated by the changes of DNA methylation in placental pathogenesis. In addition, studies are required to elucidate the distinct nature of DNA methylation in the maternal-fetal interaction during pregnancy.

\section{Acknowledgements}

The current work was supported by the Municipal Science and Technology Foundation of Qingdao (grant no. 2012-WSZD009).

\section{References}

1. Yan J, Fan L, Zhao Y, You L, Wang L, Zhao H, Li Y and Chen ZJ: DYZ1 copy number variation, Y chromosome polymorphism and early recurrent spontaneous abortion/early embryo growth arrest. Eur J Obstet Gynecol Reprod Biol 159: 371-374, 2011.

2. Luan L, Zhu X and Ma Y: Study on expression of TGF- $\beta 1$, MMP-9 and TIMP-1 in the chorionic villi of early embryo growth arrest. Pro Obstetrics Gynecol, 2012.

3. Xiao FZ and Hu ML: The prevent and reason analysis of the patients of embryo damage. China Practical Medicine 8: 27-29, 2013 (In Chinese).

4. Wang Y, Han YF, Wang ZY and Wen-Yuan LI: Clinical study on intervention measures of re-pregnancy in people with embryo damage history. Guide of China Medicine 19: 56-57, 2014 (In Chinese).

5. Bauer MK, Harding JE, Bassett NS, Breier BH, Oliver MH, Gallaher BH, Evans PC, Woodall SM and Gluckman PD: Fetal growth and placental function. Mol Cell Endocrinol 140: 115-120, 1998.

6. Beck S and Rakyan VK: The methylome: Approaches for global DNA methylation profiling. Trends Genet 24: 231-237, 2008.

7. Oda M, Yamagiwa A, Yamamoto S, Nakayama T, Tsumura A, Sasaki H, Nakao K, Li E and Okano M: DNA methylation regulates long-range gene silencing of an X-linked homeobox gene cluster in a lineage-specific manner. Genes Dev 20: 3382-3394, 2006.

8. MacDonald WA and Mann MR: Epigenetic regulation of genomic imprinting from germ line to preimplantation. Mol Reprod Dev 81: 126-140, 2014.

9. Watanabe D, Suetake I, Tada T and Tajima S: Stage- and cell-specific expression of DNMT3a and DNMT3b during embryogenesis. Mech Dev 118: 187-190, 2002.

10. Koukoura O, Sifakis $\mathrm{S}$ and Spandidos DA: DNA methylation in the human placenta and fetal growth (Review). Mol Med Rep 5: $883-889,2012$

11. O'Doherty AM, Magee DA, O'Shea LC, Forde N, Beltman ME, Mamo S and Fair T: DNA methylation dynamics at imprinted genes during bovine pre-implantation embryo development. BMC Dev Biol 15: 13, 2015.

12. Uysal F, Akkoyunlu G and Ozturk S: Dynamic expression of DNA methyltransferases (DNMTs) in oocytes and early embryos. Biochimie 116: 103-113, 2015.

13. Chen T and Li E: Structure and function of eukaryotic DNA methyltransferases. Curr Top Dev Biol 60: 55-89, 2004.

14. Yoder MC, Hiatt K, Dutt P, Mukherjee P, Bodine DM and Orlic D: Characterization of definitive lymphohematopoietic stem cells in the day 9 murine yolk sac. Immunity 7: 335-344, 1997.

15. Katari S, Turan N, Bibikova M, Erinle O, Chalian R, Foster M, Gaughan JP, Coutifaris C and Sapienza C: DNA methylation and gene expression differences in children conceived in vitro or in vivo. Hum Mol Genet 18: 3769-3778, 2009.

16. Huang K, Wu Z, Liu Z, Hu G, Yu J, Chang KH, Kim KP, Le T, Faull KF, Rao N, et al: Selective demethylation and altered gene expression are associated with ICF syndrome in human-induced pluripotent stem cells and mesenchymal stem cells. Hum Mol Genet 23: 6448-6457, 2014.

17. Ehrlich M: The ICF syndrome, a DNA methyltransferase 3B deficiency and immunodeficiency disease. Clin Immunol 109: 17-28, 2003.

18. Arand J, Wossidlo M, Lepikhov K, Peat JR, Reik W and Walter J: Selective impairment of methylation maintenance is the major cause of DNA methylation reprogramming in the early embryo. Epigenetics Chromatin 8: 1, 2015.

19. Hirasawa R and Sasaki H: Dynamic transition of Dnmt3b expression in mouse pre- and early post-implantation embryos. Gene Expr Patterns 9: 27-30, 2009. 
20. Hirasawa R, Chiba H, Kaneda M, Tajima S, Li E, Jaenisch R and Sasaki H: Maternal and zygotic Dnmtl are necessary and sufficient for the maintenance of DNA methylation imprints during preimplantation development. Genes Dev 22: 1607-1616, 2008 .

21. Livak KJ and Schmittgen TD: Analysis of relative gene expression data using real-time quantitative PCR and the 2(-Delta Delta C(T)) method. Methods 25: 402-408, 2001.

22. Spradley FT: Metabolic abnormalities and obesity's impact on the risk for developing preeclampsia. Am J Physiol Regul Integr Comp Physiol 312: R5-R12, 2017.

23. Hou SX and Dept U: Application of ultrasound in the diagnosis of intrauterine embryo growth arrest. World Latest Medicine Information 15: 29-30, 2015 (In Chinese).

24. Sharma S: Natural killer cells and regulatory $\mathrm{T}$ cells in early pregnancy loss. Int J Dev Biol 58: 219-229, 2014

25. Zobeiri F, Sadrkhanlou RA, Salami S, Mardani K and Ahmadi A: The effect of ciprofloxacin on sperm DNA damage, fertility potential and early embryonic development in NMRI mice. Vet Res Forum 3: 131-135, 2012.

26. Fuke C, Shimabukuro M, Petronis A, Sugimoto J, Oda T, Miura K, Miyazaki T, Ogura C, Okazaki Y and Jinno Y: Age related changes in 5-methylcytosine content in human peripheral leukocytes and placentas: An HPLC-based study. Ann Hum Genet 68: 196-204, 2004.

27. Maccani MA and Marsit CJ: Epigenetics in the placenta. Am J Reprod Immunol 62: 78-89, 2009.

28. Hata K, Kusumi M, Yokomine T, Li E and Sasaki H: Meiotic and epigenetic aberrations in Dnmt3L-deficient male germ cells. Mol Reprod Dev 73: 116-122, 2006.

29. Yin LJ, Zhang Y, Lv PP, He WH, Wu YT, Liu AX, Ding GL, Dong MY, Qu F, Xu CM, et al: Insufficient maintenance DNA methylation is associated with abnormal embryonic development. BMC Med 10: 26, 2012.
30. Lucifero D, La Salle S, Bourc'his D, Martel J, Bestor TH and Trasler JM: Coordinate regulation of DNA methyltransferase expression during oogenesis. BMC Dev Biol 7: 36, 2007.

31. Waterland RA and Jirtle RL: Early nutrition, epigenetic changes at transposons and imprinted genes, and enhanced susceptibility to adult chronic diseases. Nutrition 20: 63-68, 2004.

32. Robins JC, Marsit CJ, Padbury JF and Sharma SS: Endocrine disruptors, environmental oxygen, epigenetics and pregnancy. Front Biosci (Elite Ed) 3: 690-700, 2011.

33. Sood R, Zehnder JL, Druzin ML and Brown PO: Gene expression patterns in human placenta. Proc Natl Acad Sci USA 103 5478-5483, 2006.

34. Filiberto AC, Maccani MA, Koestler D, Wilhelm-Benartzi C, Avissar-Whiting M, Banister CE, Gagne LA and Marsit CJ: Birthweight is associated with DNA promoter methylation of the glucocorticoid receptor in human placenta. Epigenetics 6: 566-572, 2011.

35. Nelissen EC, van Montfoort AP, Dumoulin JC and Evers JL: Epigenetics and the placenta. Hum Reprod Update 17: 397-417, 2011.

36. Vlahović M, Bulić-Jakus F, Jurić-Lekić G, Fucić A, Marić S and Serman D: Changes in the placenta and in the rat embryo caused by the demethylating agent 5-azacytidine. Int J Dev Biol 43: 843-846, 1999.

37. Grazul-Bilska AT, Johnson ML, Borowicz PP, Minten M, Bilski JJ, Wroblewski R, Velimirovich M, Coupe LR, Redmer DA and Reynolds LP: Placental development during early pregnancy in sheep: Cell proliferation, global methylation, and angiogenesis in the fetal placenta. Reproduction 141: 529-540, 2011. 